\title{
ISOTERMAS DE ADSORÇÃO DE FREUNDLICH PARA O CRÔMIO (III) EM LATOSSOLOS'
}

\author{
Marco Aurélio Kondracki de Alcântara ${ }^{2,3,4 *}$; Otávio Antonio de Camargo ${ }^{3,5}$ \\ ${ }^{2}$ Pós-Doutorando no Centro de Solos e Recursos Agroambientais - IAC/CSRA. \\ ${ }^{3}$ IAC/CSRA, C.P. 28 - CEP: 13020-902 - Campinas, SP. \\ ${ }^{4}$ Bolsista FAPESP. \\ ${ }^{5}$ Bolsista CNPq. \\ *Autor correspondente <epska@uol.com.br>
}

\begin{abstract}
RESUMO: Os impactos causados no ambiente pela aplicação de resíduos de curtume contendo crômio em solos agrícolas devem ser estudados detalhadamente para que se evite a contaminação da biota e das águas subterrâneas. A adsorção do crômio ao solo reveste-se de especial importância em estudos de movimentação de solutos, uma vez que, se a capacidade adsortiva de um solo for ultrapassada, o metal fica potencialmente disponível para ser lixiviado. Os objetivos deste trabalho foram verificar a adequação da Isoterma de Freundlich em descrever a adsorção do crômio em Latossolos; analisar o comportamento da adsorção do crômio considerando-se os diferentes solos e horizontes, bem como de alguns de seus atributos químicos e físicos, como teores de óxidos e textura, e analisar a influência do nível de pH dos horizontes $\mathrm{A}$. Utilizaram-se amostras dos horizontes A e B de dois solos coletados no Estado de São Paulo, Brasil: a) LATOSSOLO VERMELHO ESCURO Eutroférrico (LVe) e b) LATOSSOLO VERMELHO AMARELO Distrófico (LVd). O pH das amostras do horizonte superficial foi alterado com adição de carbonato de cálcio para elevar a saturação por bases a $70 \%$. Duas repetições de cada amostra foram agitadas com soluções contendo diferentes concentrações iniciais $C_{0}$ de crômio na forma de $\mathrm{CrCl}_{3}: 0 ; 0,5 ; 1,0 ; 2,5 ; 5,0 ; 10,0$ e 50,0 $\mathrm{mg} \mathrm{L}^{-1}$. Ajustaram-se isotermas de Freundlich (exponencial) para descrever a adsorção do crômio ao solo. O modelo de Freundlich conseguiu descrever satisfatoriamente o comportamento do crômio na maioria dos tratamentos utilizados. A adsorção do crômio foi maior no LVe em relação ao LVd e foi favorecida pela calagem. Não foi possível evidenciar diferença na adsorção de crômio entre os horizontes dos dois solos estudados.
\end{abstract}

Palavras-chave: LATOSSOLO, crômio, adsorção, Freundlich

\section{FREUNDLICH ADSORPTION ISOTHERMS FOR CHROMIUM (III) IN OXISOLS}

\begin{abstract}
Environmental impacts caused by tannery waste application to agricultural soils must be studied to avoid biota and groundwater contamination. Soil chromium adsorption is very important, when the soil adsorptive capacity is exceeded and, potentially, the metals become available for leaching. The objectives of this study were to verify if the isotherm of Freundlich describes adequately the chromium adsorption by Oxisols, to study chromium adsorption considering different soils and horizons, as well as some chemical and physical attributes, and to evaluate the influence of the $\mathrm{pH}$ level on chromium adsorption. Samples of the A and B horizons of two soils were collected in the São Paulo State - Brazil: a) Typic Eutrorthox (LVe) and b) Typic Haplorthox (LVd). The pH alteration of the surface horizons was performed with the addition of calcium carbonate to reach $70 \%$ base saturation. Samples were shaken with $\mathrm{CrCl}_{3}$ solutions (prepared in $\mathrm{Ca}\left(\mathrm{NO}_{3}\right)_{2} 5 \mathrm{mmol} \mathrm{L}^{-1}$ as the support electrolyte) of different initial chromium concentrations $\mathrm{C}_{0}: 0 ; 0.5 ; 1.0$; 2.5; $5.0 ; 10.0$ and $50.0 \mathrm{mg} \mathrm{L}^{-1}$, using two replicates for each treatment. Freundlich isotherms were adjusted to describe the chromium adsorption by the soil. This isoterm fitted well to the experimental data. Chromium adsorption was higher in the Typic Eutrorthox than in the Typic Haplorthox. The adsorption was also favored by liming in both soils. There was no difference between chromium adsorption by the two horizons of the soils. Key words: Oxisols, chromium, adsorption, Freundlich
\end{abstract}

\section{INTRODUÇÃO}

A produção de couro gera resíduos com ampla variação quanto às suas características químicas. Alguns desses resíduos, como por exemplo, os lodos de curtume, podem e vêm sendo utilizados na agricultura. Esses resíduos são aplicados diretamente ao solo como fornecedores de nutrientes às plantas (principalmente $\mathrm{N}, \mathrm{Ca}, \mathrm{Mg}$ e S) e como melhoradores de outros atributos químicos e físicos. No entanto, em função da presença de crômio em alguns tipos de resíduos de curtume (naqueles provenientes da curtição do couro ao crômio, e que atualmente são os gerados em maior quantidade), os impactos causados no ambiente pela sua aplicação em solos agrícolas devem ser estudados detalhadamente para que se evite a contaminação da biota e das águas subterrâneas.

${ }^{1}$ Parte da Tese de Doutorado do primeiro autor apresentada à FEAGRI/UNICAMP - Campinas, SP. 
O crômio presente nesses resíduos encontra-se, predominantemente, na forma trivalente, de toxicidade e mobilidade muito menores em relação à hexavalente. 0 $\mathrm{Cr}$ (III) no solo é pouco móvel, e só o é em ambiente muito ácido. $\mathrm{Em} \mathrm{pH} \mathrm{5,5} \mathrm{é} \mathrm{quase} \mathrm{totalmente} \mathrm{precipitado.}$ Por isso, os seus compostos são considerados muito estáveis no solo.

A possível contaminação das águas subterrâneas pela eluviação de metais pesados depende de uma série de fatores relacionados à quantidade de resíduos colocada no solo, à concentração e solubilidade desses metais nos resíduos, ao clima local (precipitação pluvial) e à capacidade de retenção do solo (Amaral Sobrinho, 1993). As recomendações para o descarte de resíduos nos solos devem ser precedidas por estudos detalhados dos fatores que afetam a precipitação, a adsorção e o transporte desses metais. Cada solo possui uma capacidade adsortiva própria. Se essa capacidade for ultrapassada, o metal fica potencialmente disponível para ser lixiviado. Portanto, a adsorção de metais ao solo reveste-se de especial importância em estudos de movimentação de solutos.

Atualmente, existe uma tendência em concentrar os estudos de adsorção e movimentação de poluentes no solo em experimentos de laboratório, embora se reconheça que estudos de campo sejam indispensáveis ao monitoramento desses poluentes. De acordo com Fuller \& Warrick (1986), além da confiabilidade dos dados provenientes dos métodos de laboratório, essa tendência pode ser explicada pela menor duração, custo e complexidade desses experimentos em relação aos conduzidos no campo. Assim, os experimentos em laboratório exigem menor número de testes e as variações climáticas e microclimáticas são também mais facilmente controláveis.

O emprego de isotermas para medir a capacidade adsortiva de solos vem sendo realizado desde longa data. Cunha et al. (1994) citam que, inicialmente, tais isotermas eram utilizadas para explicar a adsorção de ânions, principalmente fosfato. Fuller \& Warrick (1986) comentam que os estudos de adsorção têm sido muito utilizados, entre outros motivos, quando há necessidade de reduzir as variáveis e selecionar qual a concentração mais adequada. Barrow (1978) cita dois motivos principais para se utilizarem modelos que descrevam a adsorção em solos: a) possibilitam expressar as propriedades do solo numericamente e b) permitem conhecer mais a respeito da natureza dos processos de adsorção. Diversos modelos têm sido utilizados para descrever a adsorção de elementos no solo. Entre os mais citados na literatura, encontram-se o linear, o de Freundlich (exponencial), o de Temkim (logarítmico) e o de Langmuir (Sposito, 1984).

O objetivo deste trabalho foi o de estudar a aplicação da isoterma de adsorção de Freundlich para o crômio (III) em Latossolos altamente intemperizados. Especificamente, este trabalho objetivou: (i) verificar a adequação das isotermas em descrever a adsorção do crômio nos solos e horizontes estudados; (ii) analisar o comportamento da adsorção do crômio considerando-se os diferentes solos e horizontes, bem como alguns de seus atributos químicos e físicos, como teores de óxidos e textura e (iii) avaliar a adsorção do crômio considerando-se a influência do nível de pH obtido com a calagem dos horizontes superficiais.

\section{MATERIAL E MÉTODOS}

\section{Solos}

Foram utilizados dois solos de texturas diferentes coletados no Estado de São Paulo, Brasil: a) LATOSSOLO VERMELHO ESCURO Eutroférrico (Typic Eutrorthox) - LVe, e b) LATOSSOLO VERMELHO AMARELO Distrófico (Typic Haplorthox) - LVd (EMBRAPA, 1999), que representam geograficamente importante área de disposição ou descarte de resíduos de curtume. A caracterização química e física dos solos seguiu metodologia descrita em Camargo et al. (1986). A influência do nível de $\mathrm{pH}$ foi estudada adicionando carbonato de cálcio aos dois horizontes A para elevar a saturação por bases a $70 \%$. As amostras foram incubadas por um período de três meses, permitindo a estabilização do $\mathrm{pH}$. Foram também utilizadas amostras dos horizontes A e B, sem calagem. Todas as amostras foram armazenadas mantendo-se a umidade entre 70 a $80 \%$ da capacidade de campo (umidade a $300 \mathrm{kPa}$ ).

\section{Estudos de adsorção}

A condução desse estudo seguiu metodologia adaptada de Selim et al. (1990) descrita a seguir. Inicialmente, os solos que estavam sendo armazenados úmidos foram secos ao ar e passados novamente em peneira de $2 \mathrm{~mm}$ de diâmetro. Agitaram-se $2,00 \mathrm{~g}$ de solo em agitador horizontal a 100 osc min $^{-1}$ por um período de $18 \mathrm{~h}$ em contato com $20 \mathrm{~mL}$ de soluções contendo diferentes concentrações iniciais $\mathrm{C}_{0}$ de crômio $\left(\mathrm{CrCl}_{3}\right.$ em $\left.\mathrm{Ca}\left(\mathrm{NO}_{3}\right)_{2} 5,0 \mathrm{mmol} \mathrm{L}^{-1}\right): 0 ; 0,5 ; 1,0 ; 2,5 ; 5,0 ; 10,0$ e 50,0 $\mathrm{mg} \mathrm{L}^{-1}$, utilizando-se duas repetições por tratamento. Após a agitação, as soluções sobrenadantes foram centrifugadas a $10.000 \mathrm{G}$ durante $10 \mathrm{~min}$, e então filtradas utilizando-se papel de filtro quantitativo (Whatman 42). As determinações das concentrações de crômio em equilíbrio $C_{e}$ foram feitas por espectrofotometria de emissão atômica por plasma induzido de argônio (ICP-AES).

As concentrações de crômio adsorvidas ao solo (S), foram obtidas utilizando-se a expressão: $\mathrm{S}=\left(\mathrm{C}_{0}-\right.$ $\mathrm{C}_{\mathrm{e}}$ ) FD; onde $\mathrm{S}$ é a quantidade de crômio adsorvida ao solo $\left(\mathrm{mg} \mathrm{kg}^{-1}\right)$; $\mathrm{C}_{0}$ é a concentração de crômio da solução colocada em contato com o solo $\left(\mathrm{mg} \mathrm{L}^{-1}\right) ; \mathrm{C}_{\mathrm{e}}$ é a concentração de crômio na solução após a agitação (mg $\mathrm{L}^{-1}$ ) e FD é o fator de diluição, considera a relação solução/solo ( no caso, $\mathrm{FD}=20 / 2=10$ ).

Foram feitos gráficos das concentrações adsorvidas $\mathrm{S}$ em função das concentrações em equilíbrio 
$\mathrm{C}_{e}$. Ajustaram-se, então, com auxílio do programa computacional Microcal Origin 5.0, isotermas de adsorção de Freundlich, de acordo com a expressão: $\mathrm{S}=\mathrm{K}_{\mathrm{F}} \mathrm{C}_{\mathrm{e}}{ }^{\mathrm{n}}$; onde $\mathrm{S}$ : é a massa do soluto associada à fase sólida $\left(\mathrm{mg} \mathrm{kg}^{-1}\right)$; $\mathrm{C}_{\mathrm{e}}$ é a concentração do soluto em equilíbrio na solução ( $\left.\mathrm{mg} \mathrm{L}^{-1}\right) ; \mathrm{K}_{\mathrm{F}}$ é o coeficiente de adsorção de Freundlich $\left(\mathrm{cm}^{3} \mathrm{~kg}^{-1}\right)$ e $\mathrm{n}$ a estimativa do parâmetro de ajuste (adimensional).

A discussão neste trabalho com referência à influência dos diferentes fatores nas isotermas de adsorção de crômio no solo foi feita principalmente tendo como base os coeficientes $\mathrm{K}_{\mathrm{F}}$. Paralelamente, para facilitar a visualização dos resultados em discussão, agruparam-se as curvas de adsorção em gráficos para evidenciar separadamente os efeitos dos solos, dos horizontes e da calagem na adsorção do crômio.

\section{RESULTADOS E DISCUSSÃO}

\section{Ajuste das isotermas de adsorção}

As variáveis para ajuste das isotermas de adsorção são apresentadas na TABELA 1. Para a maioria das amostras, o modelo conseguiu descrever satisfatoriamente a adsorção do crômio ao solo $\left(R^{2}\right.$ entre 0,62 e 0,87 ), com exceção do horizonte $A$ do LVd que recebeu calagem $\left(R^{2}=0,51\right)$. Cunha et al. (1994) realizaram experimentos de adsorção utilizando amostras superficiais e subsuperficiais de diversos solos, nos quais avaliaram a adequação de diferentes isotermas em descrever o comportamento da adsorção de zinco. Os autores constataram que a isoterma de Freundlich (exponencial) foi a que melhor se ajustou aos dados, seguida de perto pela de Langmuir. A de Temkim (logarítmica) proporcionou ajustes bem menos adequados. Tais resultados corroboram o uso do modelo de Freundlich para descrever a adsorção de crômio nos solos estudados.

A utilização do coeficiente de adsorção $\mathrm{K}_{F}$ permite identificar a capacidade de um solo em reter determinado soluto, possibilitando a estimativa da extensão de seu movimento na fase líquida. Este coeficiente pode ser entendido como uma medida da distribuição de equilíbrio entre as fases sólida e líquida de um solo. Assim sendo, quanto maior a capacidade adsortiva, maior será o $\mathrm{K}_{\mathrm{F}}$.

\section{Efeito dos solos na adsorção de crômio}

O LVe apresenta teores de argila superiores ao LVd (TABELA 2). A principal característica física que influencia a reatividade de um elemento no solo é a superfície específica das suas partículas. Em nossas condições a superfície específica está relacionada ao teor de argila (Grohmann, 1970), o que pode explicar, ao menos parcialmente, a maior adsorção de crômio no LVe (de textura argilosa) em relação ao LVd (de textura média).

Observa-se também na TABELA 2 que o LVe possui teores de óxidos de ferro, alumínio e manganês superiores ao $\mathrm{LVd}$ em todos os extratores utilizados
(CDB, oxalato ou $\mathrm{H}_{2} \mathrm{SO}_{4}$ ). Tais atributos podem ter sido também responsáveis pela maior capacidade adsortiva do $\mathrm{LVe}$ em relação ao $\mathrm{LVd}$. Especial atenção deve ser dada aos teores bem mais elevados de manganês facilmente reduzível do LVe. As formas $\mathrm{Mn}$ (III) e $\mathrm{Mn}$ (IV), denominadas de facilmente reduzíveis, têm importante papel na mudança do estado de oxidação do crômio. Em solos com elevados teores de manganês facilmente reduzíveis, James \& Bartlett (1983) relataram uma lenta transformação do $\mathrm{Cr}(\mathrm{III})$ à $\mathrm{Cr}(\mathrm{VI})$. Essa transformação representaria um aumento no risco de poluição do ambiente, uma vez que a forma hexavalente apresenta toxicidade, solubilidade e mobilidade muito maiores do que a forma trivalente e que foi constatada por Aquino Neto \& Camargo (2000) para este mesmo LATOSSOLO VERMELHO ESCURO.

Nas amostras sem calagem, os coeficientes de adsorção $\mathrm{K}_{\mathrm{F}}$ para o LVe e o LVd foram 63,9 e $23,6 \mathrm{~cm}^{3}$ $\mathrm{kg}^{-1}$, respectivamente. Estes valores indicam a maior capacidade adsortiva do LVe em relação ao LVd. Essa maior capacidade adsortiva pode ser facilmente visualizada observando-se as curvas de adsorção da Figura 1.

A retenção de elementos pelo solo é resultante de vários processos que interagem. Por esse motivo, correlações significativas entre os parâmetros de retenção e as propriedades dos elementos e dos solos podem ser detectadas mesmo entre solos com características muito diferentes. Buchter et al. (1989) encontraram associações entre a adsorção de crômio e os teores de determinadas formas de óxidos no solo. Esses autores estudaram a retenção de quinze elementos em onze solos de diferentes ordens objetivando determinar os efeitos das propriedades do elemento e do solo na magnitude do parâmetro $K_{F}$ da isoterma de Freundlich. Maiores quantidades de oxiânions foram retidas por solos com teores mais elevados de óxidos de ferro, alumínio e óxidos de baixa cristalinidade (extraídos com oxalato) em relação a solos

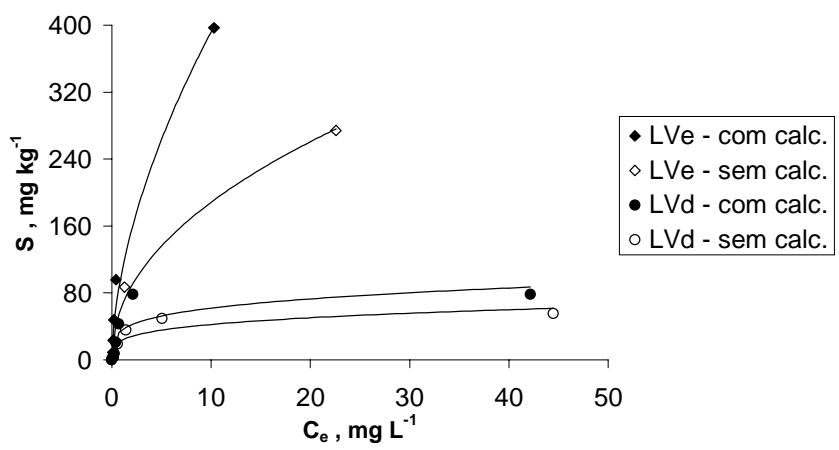

Figura 1 - Efeito dos solos e da calagem na adsorção do crômio. S: crômio adsorvido ao solo; $\mathrm{C}_{\mathrm{e}}$ : crômio na solução após a agitação; LVe: LATOSSOLO VERMELHO ESCURO Eutroférrico; LVd: LATOSSOLO VERMELHO AMARELO Distrófico; sem calc.: sem adição de carbonato de cálcio; com calc: com adição de carbonato de cálcio para elevar a saturação por bases a 70 \%. 
TABELA 1 - Coeficientes das isotermas de adsorção de Freundlich.

\begin{tabular}{lccccccc}
\hline Solo & Horizonte & calagem & \multicolumn{7}{c}{$\mathrm{K}_{\mathrm{F}}$} & $\mathrm{n}$ & $\mathrm{R}^{2}$ \\
\hline LVe & $\mathrm{A}$ & sem & $-13,9$ & $\pm 9,3$ & 0,47 & $\pm 0,05$ & 0,86 \\
& $\mathrm{~A}$ & com & 104,2 & $\pm 16,5$ & 0,57 & $\pm 0,07$ & 0,87 \\
& $\mathrm{~B}$ & sem & 78,0 & $\pm 16,7$ & 0,39 & $\pm 0,07$ & 0,78 \\
LVd & $\mathrm{A}$ & sem & 23,6 & $\pm 5,5$ & 0,25 & $\pm 0,08$ & 0,62 \\
& $\mathrm{~A}$ & com & 35,7 & $\pm 10,8$ & 0,24 & $\pm 0,10$ & 0,51 \\
& $\mathrm{~B}$ & sem & 21,5 & $\pm 3,5$ & 0,43 & $\pm 0,05$ & 0,85 \\
\hline
\end{tabular}

LVe: LATOSSOLO VERMELHO ESCURO Eutroférrico; LVd: LATOSSOLO VERMELHO AMARELO Distrófico. $\mathrm{K}_{\mathrm{F}}$ : coeficiente de adsorção de Freundlich; n: estimativa do parâmetro de ajuste da isoterma de Freundlich.

TABELA 2 - Atributos químicos e físicos dos solos utilizados.

\begin{tabular}{|c|c|c|c|c|}
\hline \multirow[b]{2}{*}{ Atributo } & \multicolumn{4}{|c|}{ Solo } \\
\hline & $\begin{array}{c}\mathrm{LVe} \\
\text { Horizonte A }\end{array}$ & $\begin{array}{c}\text { LVe } \\
\text { Horizonte B }\end{array}$ & $\begin{array}{c}\mathrm{LVd} \\
\text { Horizonte A }\end{array}$ & $\begin{array}{c}\text { LVd } \\
\text { Horizonte B }\end{array}$ \\
\hline $\mathrm{pH}$ em KCl $1 \mathrm{~mol} \mathrm{~L}^{-1}$ (original) & 4,34 & 5,26 & 3,58 & 3,93 \\
\hline pH em KCl (após a calagem) & 5,50 & - & 5,70 & - \\
\hline C orgânico $\quad\left(\mathrm{g} \mathrm{kg}^{-1}\right)$ & 15,5 & 3,8 & 8,3 & 2,9 \\
\hline Ácido Húmico " & 3,0 & 0,5 & 2,9 & 0,3 \\
\hline Ácido Fúlvico " & 3,2 & 1,4 & 1,9 & 1,2 \\
\hline Humina & 9,3 & 1,9 & 3,6 & 1,4 \\
\hline $\mathrm{Ca} \quad\left(\mathrm{mmol}_{\mathrm{C}} \mathrm{dm}^{-3}\right)$ & 13 & 18 & 4 & 4 \\
\hline $\mathrm{Mg} \quad$ " & 13 & 3 & 1 & 1 \\
\hline $\mathrm{Al}+\mathrm{H} \quad "$ & 61,3 & 27,4 & 46,4 & 22,3 \\
\hline $\mathrm{T}$ & 88 & 39 & 52 & 28 \\
\hline $\mathrm{Fe}_{2} \mathrm{O}_{3}$ em CDB $\left(\mathrm{g} \mathrm{kg}^{-1}\right)$ & 153 & 166 & 40 & 47 \\
\hline $\mathrm{Fe}_{2} \mathrm{O}_{3}$ em oxalato " & 2,0 & 3,0 & 2,0 & 1,0 \\
\hline $\mathrm{Fe}_{2} \mathrm{O}_{3}$ em H2SO4 " & 209 & 222 & 52 & 52 \\
\hline $\mathrm{Al}_{2} \mathrm{O}_{3}$ em CDB & 17 & 17 & 8 & 11 \\
\hline $\mathrm{Al}_{2} \mathrm{O}_{3}$ em oxalato & 4 & 4 & 2 & 2 \\
\hline $\mathrm{Al}_{2} \mathrm{O}_{3}$ em H $2 \mathrm{SO} 4$ & 186 & 214 & 71 & 86 \\
\hline $\mathrm{Mn}_{2} \mathrm{O}_{3}$ em CDB " & 1,2 & 0,9 & 0,4 & 0,3 \\
\hline $\mathrm{Mn}_{2} \mathrm{O}_{3}$ em oxalato " & 0,4 & 0,2 & 0,2 & 0,0 \\
\hline Mn facilm. reduzível $\left(\mathrm{mg} \mathrm{kg}^{-1}\right)$ & 146 & 45 & 1,8 & 2,5 \\
\hline Argila $\left(\mathrm{g} \mathrm{kg}^{-1}\right)$ & 560 & 580 & 120 & 150 \\
\hline Silte & 300 & 290 & 50 & 50 \\
\hline Areia & 140 & 130 & 830 & 800 \\
\hline
\end{tabular}

LVe: LATOSSOLO VERMELHO ESCURO Eutroférrico; LVd: LATOSSOLO VERMELHO AMARELO Distrófico.

com teores reduzidos desses materiais, corroborando os resultados aqui obtidos. A adsorção de crômio às diferentes formas de óxidos de $\mathrm{Fe}, \mathrm{Al}$ e $\mathrm{Mn}$ poderia explicar, ao menos em parte, a maior adsorção verificada no LVe em relação ao LVd estudado. Além dos teores diferenciados de óxidos, os maiores teores de argila do LVe em relação ao LVd podem estar contribuindo para a maior adsorção observada naquele solo.

\section{Efeito do pH dos solos na adsorção de crômio}

A calagem dos solos resultou em valores de $\mathrm{K}_{\mathrm{F}}$ mais elevados em comparação às amostras sem adição de carbonato de cálcio (TABELA 1). No LVe e no LVd, esses valores aumentaram de 63,9 para $104,2 \mathrm{~cm}^{3} \mathrm{~kg}^{-1}$ e de 23,6 para $35,7 \mathrm{~cm}^{3} \mathrm{~kg}^{-1}$, respectivamente. Tais resultados indicam que a elevação do $\mathrm{pH}$ do solo (de 4,34 para 5,50 no $L V e$ e de 3,58 para 5,70 no $L V d$ ) promoveu um aumento na adsorção de crômio. Esse favorecimento da adsorção pode ser visualizado nas curvas de adsorção apresentadas na Figura 1. Resultados similares têm sido encontrados por outros pesquisadores, corroborando os aqui obtidos. Hassan \& Garrisson (1996) conduziram experimentos de adsorção 
em três solos representativos de diferentes partes geológicas dos Estados Unidos objetivando identificar os processos que influenciam a partição de crômio na solução do solo. Os coeficientes $\mathrm{K}_{F}$ para o $\mathrm{Cr}$ (III) foram calculados para cada solo utilizando isotermas de adsorção medida após um período de 6 e de 48h, respectivamente. Os valores de $\mathrm{K}_{\mathrm{F}}$ variaram de $0,85 \mathrm{a}$ $5,6 \mathrm{~cm}^{3} \mathrm{~kg}^{-1}$. A concentração de $\mathrm{Cr}(\mathrm{III})$ na solução do solo aumentou à medida que $\mathrm{o} \mathrm{pH}$ decresceu. Os valores de $\mathrm{K}_{\mathrm{F}}$ para o $\mathrm{Cr}(\mathrm{III})$ dependem do tempo de equilíbrio em função da cinética da interconversão de espécies reagindo com o solo e da mudança resultante no total de crômio na solução com o tempo.

No LVe aqui utilizado, a calagem provocou um grande aumento na adsorção de crômio, ao passo que no LVd esse efeito não foi tão pronunciado, conforme evidenciado na Figura 1. James \& Bartlett (1983), trabalhando com adsorção e redução de crômio no solo, citam que $0 \mathrm{pH}$ altera a quantidade de cargas positivas e negativas dos colóides do solo, especialmente da matéria orgânica e dos óxidos de $\mathrm{Fe}(\mathrm{III}), \mathrm{Al}(\mathrm{III})$ e $\mathrm{Mn}(\mathrm{III}, \mathrm{IV})$. No presente estudo, tais reações poderiam estar ocorrendo em maior intensidade no LVe do que no LVd em função dos seus teores elevados desses óxidos, o que explicaria o efeito mais acentuado da calagem naquele solo.

O efeito do pH na adsorção de cátions metálicos resulta, principalmente, das mudanças na carga protônica líquida $\left(\mathrm{s}_{\mathrm{H}}\right)$ das partículas do solo. Com 0 aumento do $\mathrm{pH}, \mathrm{s}_{\mathrm{H}}$ decresce para valores mais negativos, resultando em aumento na atração eletrostática do adsorvente (solo) pelo íon metálico (crômio, com carga positiva). Ou seja, em amostras de solo reagindo com soluções contendo determinados cátions metálicos numa concentração inicial estabelecida e com valores crescentes de $\mathrm{pH}$, observa-se um aumento na quantidade de cátions adsorvidos. Isso explicaria a maior adsorção de crômio observada com o aumento do $\mathrm{pH}$ (Figura 1). Assim, o efeito foi mais pronunciado no $\mathrm{LVe}$ em relação ao $\mathrm{LVd}$, foi provavelmente decorrente dos teores mais elevados de óxidos daquele solo, conferindo um efeito de carga $\mathrm{pH}$ dependente mais pronunciado. Esse comportamento poderia ter sido diferente se outros cátions ligantes na solução estivessem competindo fortemente pelo metal, o que não deve ter ocorrido em função da aplicação de soluções contaminantes contendo somente um metal.

\section{Efeito dos horizontes dos solos na adsorção de crômio}

Para o $\mathrm{LVe}$, os valores de $\mathrm{K}_{\mathrm{F}}$ no horizonte $\mathrm{A}$ e $\mathrm{B}$ foram muito próximos $\left(63,9\right.$ e $\left.78,0 \mathrm{~cm}^{3} \mathrm{~kg}^{-1}\right)$, não diferindo conforme indicam os desvios-padrões mostrados na TABELA 1. O mesmo foi detectado no LVd (23,6 e $21,5 \mathrm{~cm}^{3} \mathrm{~kg}^{-1}$ para os horizontes A e B). As curvas de adsorção apresentadas na Figura 2 são praticamente coincidentes para os dois horizontes, mostrando não

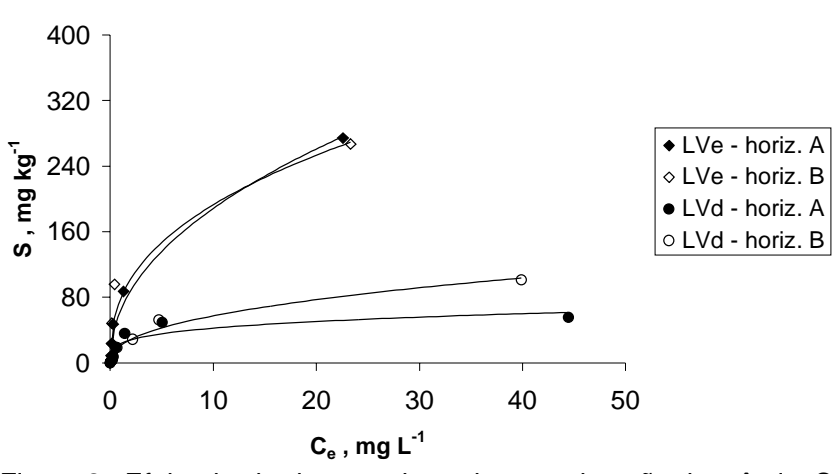

Figura 2 - Efeito dos horizontes dos solos na adsorção do crômio. S: crômio adsorvido ao solo; $C_{e}$ : crômio na solução após a agitação; LVe: LATOSSOLO VERMELHO ESCURO Eutroférrico; LVd: LATOSSOLO VERMELHO AMARELO Distrófico.

haver diferenças na adsorção de crômio no horizonte superficial em comparação com o subsuperficial. É possível que a elevada adsorção do crômio aos óxidos de $\mathrm{Fe}, \mathrm{Al}$ e $\mathrm{Mn}$ não tenha permitido evidenciar o efeito dos diferentes teores da matéria orgânica dos horizontes A e B na adsorção do metal.

\section{CONCLUSÕES}

O modelo de Freundlich conseguiu descrever satisfatoriamente a adsorção do crômio para os dois solos e horizontes.

A adsorção de crômio foi maior no LVe em relação ao $L V d$, possivelmente em decorrência dos maiores teores de óxidos e de argila daquele solo.

$\mathrm{O}$ aumento do $\mathrm{pH}$ do solo propiciado pela adição de carbonato de cálcio resultou em aumento na adsorção de crômio ao solo

Não foram detectadas diferenças na adsorção de crômio entre os horizontes superficiais e subsuperficiais dos solos estudados.

\section{AGRADECIMENTOS}

À Dra. Mônica Ferreira de Abreu e à Renata Presto, do Centro de Solos e Recursos Agroambientais do Instituto Agronômico de Campinas - IAC/CSRA - pela colaboração em várias etapas deste trabalho, em especial, nas determinações de crômio; À FAPESP e ao CNPq pela concessão das bolsas aos autores.

\section{REFERÊNCIAS BIBLIOGRÁFICAS}

AMARAL SOBRINHO, N.M.B. Interação dos metais pesados de resíduos siderúrgicos com um solo podzólico-vermelhoamarelo. Viçosa, 1993. 163p. Tese (Doutorado) - Universidade Federal de Viçosa.

AQUINO NETO, V. de; CAMARGO, O.A. Crescimento e acúmulo de crômio em alface cultivada em dois Latossolos tratados com $\mathrm{CrCl}_{3}$ e resíduos de curtume. Revista Brasileira de Ciência do Solo, v.24, p.225-235, 2000.

BARROW, N.J. The description of phosphorus adsorption curves. Journal of Soil Science, v.29, p.447-462, 1978. 
BUCHTER, B.; DAVIDOFF, B.; AMACHER, M.C.; HINZ, C.; ISKANDAR, I.K.; SELIM, H.M. Correlation of Freundlich Kd and $\mathrm{n}$ retention parameters with soils and elements. Soil Science, v.148, p.370-379, 1989.

CAMARGO, O.A. de; MONIZ, A.C.; JORGE, J.A.; VALADARES, J.M.A.S. Métodos de análise química, mineralógica e física de solos do Instituto Agronômico de Campinas. Campinas: Instituto Agronômico, 1986. 94p. (Boletim Técnico, 106).

CUNHA, R.C. de A.; CAMARGO, O.A. de; KINJO, T. Aplicação de três isotermas na adsorção de zinco em Oxissolos, Alfissolos e Ultissolos. Revista Brasileira de Ciência do Solo, v.18, p.15-20, 1994.

FULLER, W.H.; WARRICK, A.W. Soils in waste treatment and utilization. Boca Raton: CRC Press, 1986. v.2.

GROHMANN, F. A superfície específica e sua correlação com propriedades físicas e físico-químicas do solo. Piracicaba, 1970. 52p. Tese (Doutorado) - Escola Superior de Agricultura "Luiz de Queiroz", Universidade de São Paulo.
HASSAN, S.M.; GARRISON, A.W. Distribution of chromium species between soil and porewater. Chemical Speciation and Bioavailability, v.8, p.85-103, 1996.

JAMES, B.R.; BARTLETT, R.J. Behavior of chromium in soils: VII. Adsorption and reduction of hexavalent forms. Journal of Environmental Quality, v.12, p.177-181, 1983.

SELIM, H.M.; AMACHER, M.C.; ISKANDAR, I.K. Modeling the transport of heavy metals in soils. Hanover: U.S. Army Cold Regions Research and Engineering Laboratory, 1990. 155p. (Monograph 90-2).

SPOSITO, G. The surface chemistry in soils. New York: Oxford University Press, 1984. 234p.

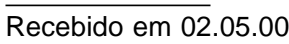

